International Journal of Wireless \& Mobile Networks (IJWMN) Vol. 3, No. 2, April 2011

\title{
SURVIVABILITY AND RELIABILITY ANALYSIS OF THE TRUSTED LINK STATE ROUTING PROTOCOL FOR WIRELESS AD HOC NETWORKS
}

\author{
Eyad Taqieddin ${ }^{1}$, Ann Miller ${ }^{2}$, and S. Jagannathan ${ }^{2}$ \\ ${ }^{1}$ Department of Network Engineering and Security, Jordan University of Science and \\ Technology, Irbid, Jordan \\ eyadtaqejust.edu.jo \\ ${ }^{2}$ Department of Electrical and Computer Engineering, Missouri University of Science \\ and Technology, Rolla, MO, USA \\ milleran@mst.edu, sarangap@mst.edu
}

\begin{abstract}
The nodes in a wireless network are responsible for both sending their traffic as well as relaying the traffic of other nodes in the network. This form of collaboration between the nodes is essential for the proper delivery of data. Without fair participation of all nodes in the routing process, some nodes may lose their energy reserves at a high rate compared to other nodes in the network. However, bandwidth and energy are not the only issues in wireless networks; survivability and reliability are critical as well.

Our focus in this work is on two link state routing protocols; OLSR and TLR. We study the effect of using these protocols on the survivability and the reliability of wireless networks. Both analytical and simulation work show that TLR results in better performance due to the inherent energy aware approach and the traffic partition used to reduce congestions in the network.
\end{abstract}

\section{KEYWORDS}

Reliability, Survivability, Energy, Delay, Wireless Networks.

\section{INTRODUCTION}

In mobile ad-hoc networks, a set of nodes collaborate with each other to guarantee proper communication between any pair of nodes in the network. This is done with no centralized entities to monitor the operation. Due to the dynamic topology, the links in the network are established and broken continuously depending on the velocity, direction and transmission range of the nodes. Unlike wired networks, the nodes in an ad hoc network have no fixed infrastructure and are limited in communication range. Intermediate nodes need to forward the packets in the case where the destination node is not within the transmission range of the source.

Efficient routing in ad hoc networks is needed to properly deliver the packets to their intended destinations with minimal delay and to extend the operational lifetime of the network through energy efficient selection of the routes.

Many energy aware routing algorithms were proposed with the aim of improving the survivability of the network by routing packets from their sources to the destinations with the minimal amount of energy. The Minimum Battery Cost Routing (MBCR) is presented in [8]. This algorithm selects the routes with the maximum total capacity. As the name implies, the routes with the least battery cost among all candidate routes are selected. Another algorithm is Simple Energy Aware Dynamic Source Routing (SEADSR) [3]. This is an extension of DSR in 
International Journal of Wireless \& Mobile Networks (IJWMN) Vol. 3, No. 2, April 2011

which a node, upon receiving the RREQ, incorporates a delay that is proportional to its energy level. This is done because DSR, by design, does not use cost functions in route selection. The authors in [5] present the Optimal Energy-Delay Routing protocol (OEDR), an algorithm based on finding a tradeoff between delay and energy consumption in LSR and compares that to OLSR. Paths are selected based on the least incurred delay and energy consumed. The Power Aware Multi-Access with Signaling (PAMAS) [6] is a protocol that efficiently uses energy by powering off nodes that are not sending or receiving packets. In [1], a detailed analysis of the network behavior both in steady and transient states is given. When the routing nodes move out of the range, then the packets in transit will either be dropped or delayed and this affects the performance of the network. Another analysis that is given in [9] studies the effect of mobility on the availability of the paths. Both [1] and [9] use a the Continuous Time Markov Model (CTMC) to model the connection availability for a two hop ad hoc network with respect to node mobility and failures.

In [7], we proposed a Link State Routing protocol based on the Optimized Link State Routing (OLSR) [4]. Our proposed protocol, the Trust Levels Routing protocol (TLR), adds security measures to counter the vulnerabilities in OLSR and to better consume the energy resources of the nodes in the network. In this work, we will analyze both OLSR and TLR in terms of survivability and reliability to emphasize the improved performance of the network in terms of lifetime and proper delivery of data. Our focus is to explain analytically the achieved improvements in the energy consumption and its effect on the survivability and reliability. The analysis is further supported by simulation scenarios.

The paper is organized as follows, in section 2 we present an overview of OLSR and TLR. Sections 3 gives a discussion of the survivability analysis of TLR followed by the reliability analysis in section 4. Simulation results are given in section 5 and the paper is concluded in section 6 .

\section{OVERVIEW OF OLSR AND TLR}

The routing protocol should focus on energy conservation to increase the lifetime of the nodes while choosing routes with the least delay, jitter and congestion. Occasionally, the best routes for several sources, in terms of delay, go through the same node whose energy gets consumed at a higher rate compared to other nodes. This, eventually, leads to a premature loss of the battery of the node. A more efficient approach is to route packets through paths that may have higher delays but with more energy resources in order to extend the lifetime of the network.

OLSR and TLR are proactive link state routing protocols. Their operation is table driven through periodically exchanging topology information with other nodes in the network. The cornerstone in the operation of both protocols is the use of Multi Point Relay (MPR) nodes which limit the flooding in the network to a specific set of nodes that are chosen according to the dynamics of the network. In OLSR, the algorithm searches for the smallest set of MPR nodes, i.e. it selects the nodes that cover the largest number of two hop neighbors. As for TLR, the MPR set is selected based on a tradeoff between the delay incurred, energy consumed and load balancing as will be explained later. In addition to energy conservation, TLR provides guarantees of the integrity, timeliness and authenticity of the packets. Our proposed protocol uses the following metrics when considering the selection of the MPR nodes.

\subsection{Energy consumed per packet}

For this metric, the best path is selected based on the least consumed total energy. Any packet going from source $n_{1}$ to destination $n_{k}$ through some intermediate nodes will consume 


$$
E_{t}=\sum_{i=1}^{k-1}\left(e^{t}{ }_{(i, i+1)}+e^{r}{ }_{(i, i+1)}\right)
$$

where $\mathbf{E}_{\mathbf{t}}$ is the total energy consumed and $\mathbf{e}^{\mathbf{t}}(\mathrm{i}, \mathrm{i}+1)$ is the energy consumed to send the packet from $n_{i}$ to $n_{i+1}$ and $\mathbf{e}^{r}(i, i+1)$ is the energy consumed by $n_{i+1}$ when receiving a packet from $n_{i}$.

\subsection{Delay per packet}

Similar to the energy metric, the goal is to find the path with the least total delay. For a packet going from node $n_{1}$ to node $n_{k}$ through some intermediate nodes, the total delay incurred is given by

$$
D_{t}=\sum_{i=1}^{k-1} d_{(i, i+1)}
$$

where $\mathbf{D}_{\mathbf{t}}$ is the total delay and $\mathrm{d}(\mathrm{i}, \mathrm{i}+1)$ is the time that starts when a packet enters the queue of node $n_{i}$ until it reaches the queue of $n_{i+1}$. Note that a trade off between the two metrics exists.

\subsection{Residual energy levels}

This metric is used to guarantee that all nodes will have approximately equal rates of consumption by using the nodes with the highest energy levels. That is, the lower the remaining available energy in a node, the higher the cost of routing through it. The cost in this case can be taken as the reciprocal of the residual energy.

\subsection{Traffic partition}

This metric serves multiple purposes. First, less congestion and delay will be incurred through the intermediate nodes. Second, a higher throughput will be achieved because data packets are going through different paths. More importantly, the traffic will be fragmented into multiple paths which can potentially reduce the ability of a malicious node to capture the whole stream of traffic.

Since TLR bases the selection of MPRs on a composite metric that differs from that of OLSR, the MPR set chosen does not necessarily have to be identical for the same network topology and conditions. Moreover, if the nodes are stationary, the MPR set for nodes running OLSR will always be the same until one or more nodes lose their battery power. In TLR, the MPR set is dynamically changed more frequently based on the MPR routing criteria.

TLR is enforced with security mechanisms to overcome some of the security threats that may impede the operation of the network. The control packets are signed and the data stream is encrypted with using a lightweight encryption algorithm.

\subsection{MPR Selction Algorithm}

The following notation will be used

$\mathbf{N}$ : set of nodes in network

s: source node

d: destination node

$\mathbf{N}_{\mathbf{1}}(\mathbf{s})$ : One-hop neighbors of node $\mathrm{s}$ 
$\mathbf{N}_{\mathbf{2}}$ (s): Two-hop neighbors of node $\mathrm{s}$

$\operatorname{MPR}(\mathbf{s}):$ The set of nodes selected as MPRs by node $\mathrm{s}\left(\mathrm{MPR}(\mathrm{s}) \in \mathrm{N}_{1}(\mathrm{~s})\right)$

$\mathbf{P}_{\mathbf{x}, \mathbf{y}}$ : Number of packets sent from $x$ through MPR $y$.

$\mathbf{w}_{1}$ and $\mathbf{w}_{2}$ : Weight factors

$\mathbf{C}_{\mathbf{x}, \mathbf{y}}$ : cost of link between nodes $\mathrm{x}$ and $\mathrm{y}$, where

$$
\mathrm{C}_{\mathrm{x}, \mathrm{y}}=\mathrm{w}_{1} *\left(\text { Energy }_{\mathrm{y}} * \text { Delay }_{\mathrm{y}}\right)+\mathrm{w}_{2} * \mathrm{P}_{\mathrm{x} y}
$$

Initially the MPR set MPR(s) is empty.

1. First, find all the nodes in $\mathrm{N}_{2}(\mathrm{~s})$ that have a single neighbor in $\mathrm{N}_{1}(\mathrm{~s})$. Add these nodes of $\mathrm{N}_{1}(\mathrm{~s})$ to the MPR set if they are not already in MPR(s). (Because there are no other MPR candidates).

2. While there exists a node in $\mathrm{N}_{2}(\mathrm{~s})$ for which MPR node is not selected, do:

a. TLR: for each node in $\mathrm{N}_{2}(\mathrm{~s})$, with multiple neighbors from $\mathrm{N}_{1}(\mathrm{~s})$, select a neighbor from $\mathrm{N}_{1}(\mathrm{~s})$ as an MPR node which results in minimum cost from $\mathrm{s}$ to the node in $\mathrm{N}_{2}(\mathrm{~s}), \mathrm{C}_{\mathrm{MPR}}$ according to (3), and add it to the MPR set if is not already in MPR(s)

b. OLSR: for each node in $\mathrm{N}_{2}(\mathrm{~s})$, with multiple neighbors from $\mathrm{N}_{1}(\mathrm{~s})$, select a neighbor from $\mathrm{N}_{1}(\mathrm{~s})$ as an MPR node which covers the maximum number of two hop neighbors in $\mathrm{N}_{2}(\mathrm{~s})$ and add it to the MPR set if it is not already in $\operatorname{MPR}(\mathrm{s})$.

\section{SURVIVABILITY ANALYSIS OF TLR}

The survivability of the network is directly related to the lifetime of nodes in the network. Clearly, the energy in every node in the network has to be properly utilized in order to avoid premature depletion of energy of the nodes and to minimize the variance in energy levels between them. The power consumption is due to 1) transmission of a packet; 2) reception of a packet 3) retransmission of packets due to congestion 4) power used when a node is idle.

Given a network modelled as a graph $\mathrm{G}=(\mathrm{N}, \mathrm{L})$ where $\mathrm{N}$ is the set of nodes and $\mathrm{L}$ is the set of links $(i, j) \in N$. A link $(i, j)$ exists if and only if $j \in N_{1}(i)$. The lifetime of a node in the network running the OLSR protocol is given as

$$
L T_{i}=\frac{E i}{(1+\gamma) \sum_{k \in \mathrm{N} 1(\mathrm{i})} e_{t x} r_{i \rightarrow k}+\sum_{j: i \in \mathrm{N} 1(\mathrm{j})} e_{r x} r_{j \rightarrow i}+\mathcal{E}}
$$

where,

Ei: Energy level of node i at $\mathrm{t}=0$ (Joule)

$\mathrm{e}_{\mathrm{tx}}$ : Energy consumed to transmit data (Joule/bit)

$\mathrm{e}_{\mathrm{rx}}$ : Energy consumed to receive data (Joule/bit)

$r_{i} \rightarrow j$ : Data rate between source $\mathrm{i}$ and destination $\mathrm{j}(\mathrm{bit} / \mathrm{sec})$ 
$\varepsilon$ : Energy consumed during the idle time (Joule/sec)

$\gamma$ : Factor representing the needed retransmissions

As for a node running TLR, the lifetime is

$$
L T_{i}=\frac{E i}{\left(\alpha+\beta \gamma \sum_{k \in \mathrm{N} 1(\mathrm{i})} e_{t x} r_{i \rightarrow-k}+\sum_{j: i \in \mathrm{N} 1(\mathrm{j})} e_{j \rightarrow i} r_{j \rightarrow}+\mathcal{E}+\mu\right.}
$$

with

$\mu$ : Energy used for overhead operations in TLR (encryption functionality).

$\alpha$ and $\beta$ : Factors comprising the overall number of packets transmitted.

The total energy used for the transmission of packets and the total energy to compensate for repeated packets due to congestion are multiplied by factors $\alpha$ and $\beta$ (both less than 1), respectively. This is a result of the load balancing in TLR which results in a node being used less frequently compared to the case of OLSR. Moreover, load balancing results in less congestion leading to fewer dropped packets. Note that the factors $\alpha$ and $\beta$ are inversely proportional to the number of available MPR candidates.

TLR outperforms OLSR in terms of energy conservation by distributing the load on various intermediate nodes. This leads to a relatively smaller variance in the battery levels and longer lifetime for the whole network.

To illustrate, consider the network given in Figure 1 where nodes S1 and S2 send traffic through nodes A, B and C to the destinations D1 and D2, respectively. Assuming that each of nodes S1 and S2 has 50 packets to send and also assume that each of nodes A, B and C has sufficient energy to receive and forward 100 packets only. With OLSR, the node that covers the maximum number of hops will be selected as the MPR. In this case, node $\mathrm{C}$ will be selected because it covers 2 nodes (D1 and D2) whereas nodes A and B only cover D1 and D2, respectively. Thus, both nodes $\mathrm{S} 1$ and $\mathrm{S} 2$ will send all their traffic through $\mathrm{C}$ which will result in consuming all it energy resources after sending 50 each (100 in total). After that, S1 will select A as the MPR whereas S2 will select B. In this scenario, node C lost all its energy prematurely whereas nodes A and B still maintained their initial energy reserves. If either node A or B moves out range then network will be partitioned due to the lack of available paths.

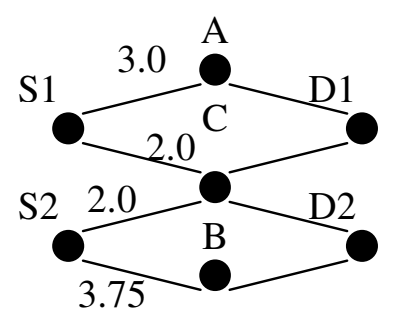

Figure 1. Ad hoc network with seven nodes.

On the other hand, when TLR is used with the same network topology using the initial link costs given in figure 1 , node $\mathrm{C}$ will be initially selected as the MPR because it has the least cost 
for both S1 and S2. After forwarding 20 packets, the link cost for S1-C and S2-C will be 3.5. Thus, on the next topology update, S1 will switch to node A for forwarding whereas S2 will maintain the same link S2-C. After transmitting 20 more packets, the updated link costs will be ( $\mathrm{S} 1-\mathrm{A}=4.33, \mathrm{~S} 1-\mathrm{C}=2.86, \mathrm{~S} 2-\mathrm{C}=3.86, \mathrm{~S} 2-\mathrm{B}=3.75$. As a result, node $\mathrm{S} 1$ will use the link S1-C and node S2 will use S2-B. Table 1 details the energy levels of the nodes throughout the above mentioned scenario. Note that node $\mathrm{C}$ still retains $40 \%$ of its energy whereas it consumed all its energy when OLSR was used.

Table 1. Remaining energy levels of the three routing nodes using TLR

\begin{tabular}{|c|c|c|c|c|c|c|c|c|c|}
\hline \multirow{2}{*}{$\begin{array}{c}\# \\
\text { Packets } \\
\text { Sent }\end{array}$} & \multicolumn{4}{|c|}{ Link Costs } & \multicolumn{2}{|c|}{ Active Link } & \multicolumn{3}{|c|}{ Remaining Energy } \\
\hline & S1-A & S1-C & S2-C & S2-B & S1 & S2 & $\mathbf{A}$ & B & $\mathbf{C}$ \\
\hline 0 & 3.0 & 2.0 & 2.0 & 3.75 & S1-C & S2-C & $100 \%$ & $100 \%$ & $100 \%$ \\
\hline 20 & 3.0 & 3.5 & 3.5 & 3.75 & S1-A & S2-C & $100 \%$ & $100 \%$ & $80 \%$ \\
\hline 40 & 4.33 & 2.86 & 3.86 & 3.75 & S1-C & S2-B & $90 \%$ & $100 \%$ & $70 \%$ \\
\hline 60 & 3.33 & 4.333 & 3.33 & 5.17 & S1-A & S2-C & $90 \%$ & $90 \%$ & $60 \%$ \\
\hline 80 & 4.75 & 4.0 & 5.0 & 4.17 & S1-C & S2-B & $80 \%$ & $90 \%$ & $50 \%$ \\
\hline 100 & 3.75 & 6.0 & 5.0 & 5.69 & S1-A & S2-C & $80 \%$ & $80 \%$ & $40 \%$ \\
\hline
\end{tabular}

\section{RELIABILITY ANALYSIS OF TLR}

The reliability of a system is defined as the conditional probability that the system is operational at time $t$ given that it was operational at time $\mathrm{t}_{0}$. Similarly, the reliability of a routing protocol can be given as the conditional probability that the network will properly deliver the packets from the source to the destination given that the routing paths were available at time $\mathrm{t}_{0}$.

Considering that a network is a system consisting of nodes and links then the reliability of the network will be directly dependent on the reliability of the components. Any failure to either of the nodes or links may lead to disruption of the service of the network. The service may remain available due to the redundancy of paths through various nodes. In this section, we analyze the reliability of OLSR and TLR for stationary nodes using Markov analysis.

\subsection{Markov Analysis}

Markov analysis provides a way of studying the sequence of events that a system may execute. This sequence of random and related events is useful in determining the reliability and availability of the system since it reflects the transition probabilities between the system states.

A Markov model is a function of two random variables; the time of observation and the current state, irrespective of the previous sequence that led to the current state.

The main advantages using Markov analysis are the simplicity of constructing the model, availability of transition probabilities between states and the incorporation of component redundancy. However, one drawback of this form of analysis is that the complexity increases with the number of states.

A Continuous Time Markov Chain (CTMC) can be used to model a system with a finite number of states to obtain a closed form solution of the reliability or availability of the system. For simplicity, we will assume that 1) component failures are mutually independent; 2) failures occur at an exponential rate; 3) equal repair rate for all component failures and 4) only one 
failure can occur during transition between states. The last condition is to guarantee that no two routers fail during a switching delay period.

Consider the Markov reliability model given in figure 3. The probability of transition from state $\mathrm{S}_{0}$ to $\mathrm{S}_{1}$ in time $\Delta \mathrm{t}$ is equal to $\lambda_{0} \Delta \mathrm{t}$ and the probability of remaining the same state is (1- $\left.\lambda 0\right) \Delta \mathrm{t}$. We can find the probabilities of the system being in either of the states at time $\mathrm{t}=\mathrm{t}+\Delta \mathrm{t}$ using the difference equations.

$$
\begin{aligned}
& \mathrm{P}_{\mathrm{S} 0}(t+\Delta t)=\left(1-\lambda_{0} \Delta t\right) \mathrm{P}_{\mathrm{S} 0}(t) \\
& \mathrm{P}_{\mathrm{S} 1}(t+\Delta t)=\left(\lambda_{0} \Delta t\right) \mathrm{P}_{\mathrm{S} 0}(t)+\left(1-\lambda_{1} \Delta t\right) \mathrm{P}_{\mathrm{S} 1}(t) \\
& \mathrm{P}_{\mathrm{S} 2}(t+\Delta t)=\left(\lambda_{1} \Delta t\right) \mathrm{P}_{\mathrm{S} 1}(t)+\left(1-\lambda_{2} \Delta t\right) \mathrm{P}_{\mathrm{S} 2}(t) \\
& \mathrm{P}_{\mathrm{S} 3}(t+\Delta t)=\left(\lambda_{2} \Delta t\right) \mathrm{P}_{\mathrm{S} 2}(t)+\mathrm{P}_{\mathrm{S} 3}(t)
\end{aligned}
$$

Rearranging the equations:

$$
\begin{aligned}
& \frac{P_{S 0}(t+\Delta t)-P_{S 0}(t)}{\Delta t}=-\lambda_{0} \mathrm{P}_{\mathrm{S} 0}(t) \\
& \frac{\mathrm{P}_{\mathrm{S1}}(t+\Delta t)-P_{S 1}(t)}{\Delta t}=\lambda_{0} P_{S 0}(t)-\lambda_{1} P_{S 1}(t) \\
& \frac{\mathrm{P}_{\mathrm{S2}}(t+\Delta t)-P_{\mathrm{S} 2}(t)}{\Delta t}=\lambda_{1} P_{S 1}(t)-\lambda_{2} P_{S 2}(t) \\
& \frac{\mathrm{P}_{\mathrm{S3}}(t+\Delta t)-P_{\mathrm{S3}}(t)}{\Delta t}=\lambda_{2} P_{S 2}(t)
\end{aligned}
$$

When $\Delta t \rightarrow 0$

$$
\begin{aligned}
& \frac{d P_{S 3}(t)}{d t}=\lambda_{2} P_{S 2}(t) \\
& \frac{d P_{S 2}(t)}{d t}=\lambda_{1} P_{S 1}(t)-\lambda_{2} P_{S 2}(t) \\
& \frac{d P_{S 1}(t)}{d t}=\lambda_{0} P_{S 0}(t)-\lambda_{1} P_{S 1}(t) \\
& \frac{d P_{S 0}(t)}{d t}=-\lambda_{0} P_{S 0}(t)
\end{aligned}
$$

The set of differential equations (14)-(17) can be solved to find the overall reliability as will be shown in section 4.2.

The analysis will use the CTMC to find the reliability of a network consisting of stationary nodes with no repair of dead nodes.

\subsection{Reliability Analysis}

The reliability of any ad hoc network depends on the reliability of the nodes. Thus, the failure of the network will be proportional to the expected lifetime of the nodes in the network. The 
network reliability and survivability calculation, however, is not simple; at some point, given the topology and the number/location of failed nodes, a network will fail. This will be case/state specific. However, we can make the following generalizations. The failure rate, $\lambda$, is defined as the number of failures per unit of time. One of the advantages of TLR is that it increases the average lifetime of all the nodes and decreases the variance of energy levels between the nodes compared to OLSR. In effect, TLR decreases the failure rate of the nodes which results in higher reliability.

By design, OLSR runs the nodes in a standby mode where only one node is responsible of routing and all other nodes remain in idle mode. When the routing node dies, another node will be selected to perform the routing. On the other hand, TLR runs the nodes in a parallel mode where one node handles the routing functionality for some time then another node takes over. The routing nodes in such a scenario will have a longer lifetime as was shown in (5).

Consider the network given in figure 2, in which S1 has no direct access to its two hop neighbor D1. Instead, nodes A, B and C will be used to route the traffic.

For OLSR, the reliability Markov model is given in figure 3. The system may be in one of the four states $\{\mathrm{Si} \mid \mathrm{i}=0,1,2,3\}$. State $\mathrm{S}_{0}$ represents the initial state of the network at time $\mathrm{t}=0$ and all three nodes will be functional whereas $S_{3}$ is the state when all three routing nodes fail.

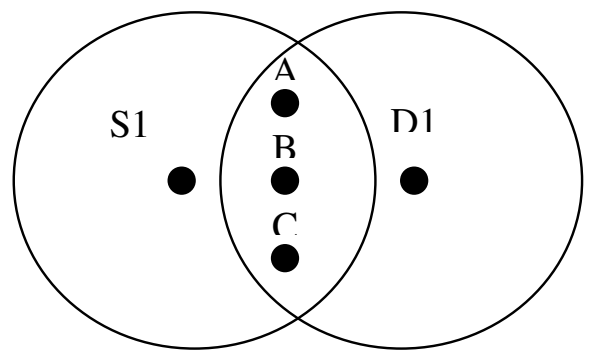

Figure 2. Ad hoc network with five nodes.

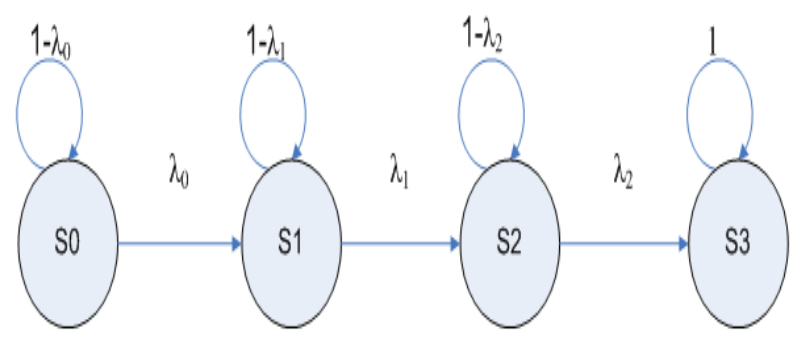

Figure 3. Markov Reliability Model for the network running OLSR

The probabilities of each of the states can be calculated using equations. (14) -(17) with the initial conditions $\mathrm{P}_{\mathrm{S} 0}(0)=1, \mathrm{P}_{\mathrm{S} 1}(0)=\mathrm{P}_{\mathrm{S} 2}(0)=\mathrm{P}_{\mathrm{S} 3}(0)=0$. Taking the Laplace transform of the above equations yields

$$
\begin{aligned}
& s P_{S 3}(s)=\lambda_{2} P_{S 2}(s) \\
& s P_{S 2}(s)=\lambda_{1} P_{S 1}(s)-\lambda_{2} P_{S 2}(s)
\end{aligned}
$$


International Journal of Wireless \& Mobile Networks (IJWMN) Vol. 3, No. 2, April 2011

$$
\begin{aligned}
& s P_{S 1}(s)=\lambda_{0} P_{S 0}(s)-\lambda_{1} P_{S 1}(s) \\
& s P_{S 0}(s)-1=-\lambda_{0} P_{S 0}(s)
\end{aligned}
$$

Rearranging the equations

$$
\begin{aligned}
& P_{S 0}(s)=\frac{1}{s+\lambda_{0}} \\
& P_{S 1}(s)=\frac{\lambda_{0}}{\left(s+\lambda_{0}\right)\left(s+\lambda_{1}\right)} \\
& P_{S 2}(s)=\frac{\lambda_{0} \lambda_{1}}{\left(s+\lambda_{0}\right)\left(s+\lambda_{1}\right)\left(s+\lambda_{2}\right)}
\end{aligned}
$$

Converting back to the time domain

$$
\begin{aligned}
& P_{S 0}(t)=e^{-\lambda_{0} t} \\
& P_{S 1}(t)=\frac{\lambda_{0}}{\left(\lambda_{1}-\lambda_{0}\right)}\left[e^{-\lambda_{0} t}-e^{-\lambda_{1} t}\right] \\
& P_{S 2}(t)=\lambda_{0} \lambda_{1}\left[\frac{e^{-\lambda_{0} t}}{\left(\lambda_{1}-\lambda_{0}\right)\left(\lambda_{2}-\lambda_{0}\right)}+\frac{e^{-\lambda_{1} t}}{\left(\lambda_{0}-\lambda_{1}\right)\left(\lambda_{2}-\lambda_{1}\right)}+\frac{e^{-\lambda_{2} t}}{\left(\lambda_{0}-\lambda_{2}\right)\left(\lambda_{1}-\lambda_{2}\right)}\right]
\end{aligned}
$$

If $\lambda_{0}=\lambda_{1}=\lambda_{2}=\lambda$ then we can find $\mathrm{P}_{\mathrm{S} 1}(\mathrm{t})$ and $\mathrm{P}_{\mathrm{S} 2}(\mathrm{t})$ using L'hospital's rule (ordinary substitution would not work because the equations would yield $0 / 0$ ).

$$
\begin{aligned}
& P_{S 0}(t)=e^{-\lambda t} \\
& P_{S 1}(t)=\lambda t e^{-\lambda t} \\
& P_{S 2}(t)=\frac{(\lambda t)^{2}}{2} e^{-\lambda t}
\end{aligned}
$$

The network will remain operational as long as it remains in $S_{0}, S_{1}$, or $S_{2}$. Thus, the reliability of the network will be

$$
R(t)=P_{S 0}(t)+P_{S 1}(t)+P_{S 2}(t)=e^{-\lambda t}+\lambda t e^{-\lambda t}+\frac{(\lambda t)^{2}}{2} e^{-\lambda t}
$$

An interesting observation is that the terms in $\mathrm{R}(\mathrm{t})$ are the first three terms in the Poisson distribution. It can be read as the probability of zero failures in time t plus the probability of one failure in time $\mathrm{t}$ plus the probability of 2 failures in time $\mathrm{t}$. Extending the model to $\mathrm{n}$ nodes would result in

$$
R(t)=\sum_{i=0}^{n-1} \frac{(\lambda t)^{i}}{i !} e^{-\lambda t}
$$


Figure 4 shows the reliability Markov model for the TLR protocol. Here, the transition probability from state $S_{0}$ to $S_{1}$ is $3 \lambda \Delta t$ because all three nodes are functional and anyone of them could fail. The same logic can be applied to the other transitions in the figure.

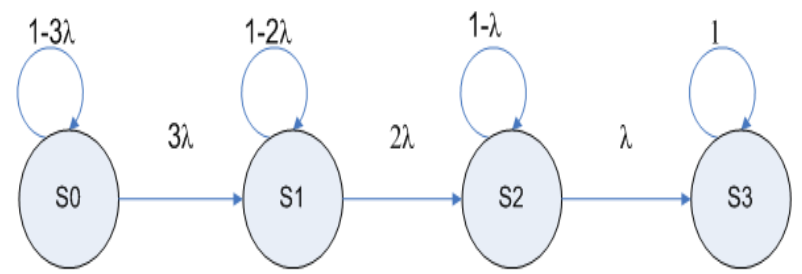

Figure 4. Markov Reliability Model for the network running TLR

The reliability can be either calculated in a similar manner to that done for OLSR or we can use the fact that all the components are working in parallel and the system will be functional if either one of them is working.

$$
\mathrm{R}(\mathrm{t})=\mathrm{P}(\mathrm{A}+\mathrm{B}+\mathrm{C})=3 e^{-\lambda t}-3 e^{-2 \lambda t}+e^{-3 \lambda t}
$$

The reliability of a network with $\mathrm{n}$ routing nodes can be given as

$$
\begin{aligned}
\mathrm{R}(\mathrm{t})= & \sum_{\mathrm{i}=1}^{\mathrm{n}} \mathrm{P}\left(\mathrm{A}_{\mathrm{i}}\right)-\sum_{\mathrm{i}=1}^{\mathrm{n}} \sum_{\mathrm{j}=\mathrm{i}+1}^{\mathrm{n}} \mathrm{P}\left(\mathrm{A}_{\mathrm{i}} \mathrm{A}_{\mathrm{j}}\right) \\
& +\sum_{i=1}^{n} \sum_{j=i+1 k=j+1}^{n} \sum_{\mathrm{i}}^{n} \mathrm{P}\left(\mathrm{A}_{\mathrm{i}} \mathrm{A}_{\mathrm{j}} \mathrm{A}_{\mathrm{k}}\right)-\ldots \\
& +(-1)^{\mathrm{n}-1}\left[\mathrm{P}\left(\mathrm{A}_{1} \mathrm{~A}_{2} \ldots \mathrm{A}_{\mathrm{n}}\right)\right]
\end{aligned}
$$

Looking back at (5), we see the lifetime of a node will increase as the factors $\alpha$ and $\beta$ decrease. Evidently, with more MPR candidate these factors must decrease since the node will be forwarding or retransmitting fewer packets. When only one MPR is available then $\alpha$ and $\beta$ will be equal to 1.0 as the packets must follow the same path.

The relation between the failure rates for nodes running TLR $\left(\lambda_{\text {TLR }}\right)$ and that of OLSR $\left(\lambda_{\text {OLSR }}\right)$ is

$$
\lambda_{T L R}=\left[\frac{(\alpha+\beta \gamma) \sum_{k \in \mathrm{N} 1(\mathrm{i})} e_{t x} r_{i->k}+\sum_{j: i \in \mathrm{N} 1(\mathrm{i})} e_{r x} r_{j \rightarrow i}+\varepsilon+\mu}{(1+\gamma) \sum_{k \in \mathrm{N} 1(\mathrm{i})} e_{t x} r_{i \rightarrow k}+\sum_{j: i \in \mathrm{N} 1(\mathrm{j})} e_{r x} r_{j \rightarrow i}+\varepsilon}\right] \lambda_{\text {OLSR }}
$$

To illustrate, assume that the failure rate of nodes resulting from running the OLSR and TLR protocol are $\lambda_{\text {OLSR }}=0.05$ and $\lambda_{T L R}=0.025$, respectively. The reliability functions of the network for both protocols are given in figure 5 . 
If the parameters of the network such as the congestion and data rates are known then we can determine the suitable encryption algorithm to be used based on the value of $\mu$, according to the following condition

$$
\mu<((1-\alpha)+\gamma(1-\beta)) \sum_{k \in \mathrm{N} 1(\mathrm{i})} e_{t x} r_{i->k}
$$

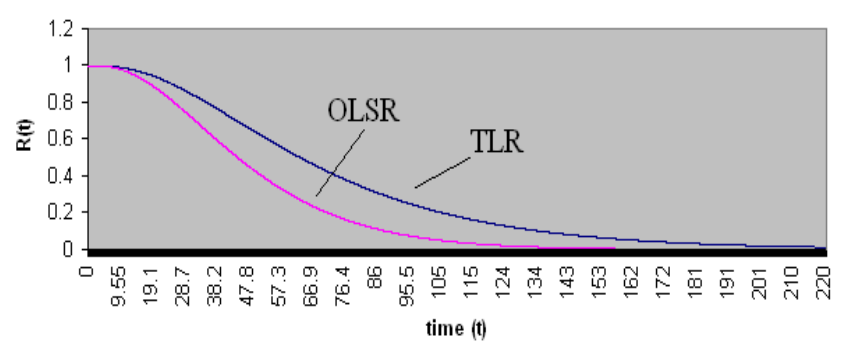

Figure 5. Reliability curves for OLSR and TLR

\section{Simulation RESUltS}

We used the network simulator NS2 to study the effect of running TLR on the survivability of the nodes in the network. We implemented the algorithm as an extension to the existing OLSR code by modifying the selection of the MPR nodes in NS2. The energy values for transmission were increased to simulate the extra energy needed for encryption. The simulations were conducted in a 670 x 670 area with 100 nodes. The nodes communicate using Constant Bit Rate (CBR) sources that are connected to the nodes in a random fashion. The data rates of 64,128 , 256 and $512 \mathrm{kpbs}$ were used and the results of the runs were averaged. Each run had a period of 100 seconds.

The first objective of the simulation was to show the distribution of the energy in the nodes after running for the whole simulation period. After each run, the remaining energy of each node was sorted into one of 10 categories based on the percentage of the remaining energy compared to the energy level at the beginning of the simulation.

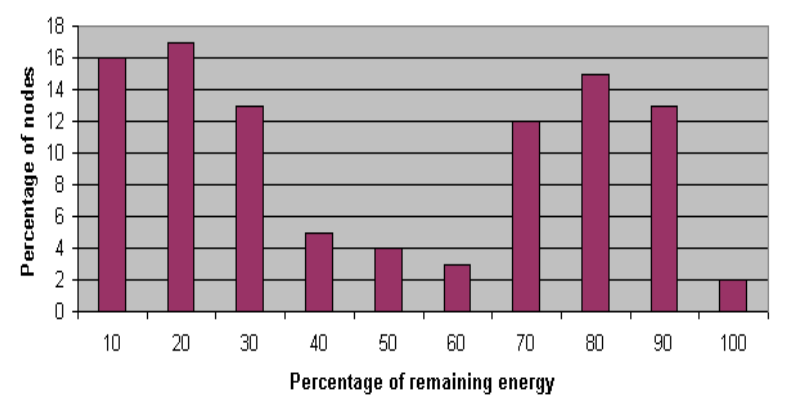

Figure 6. Energy distribution in nodes running OLSR 


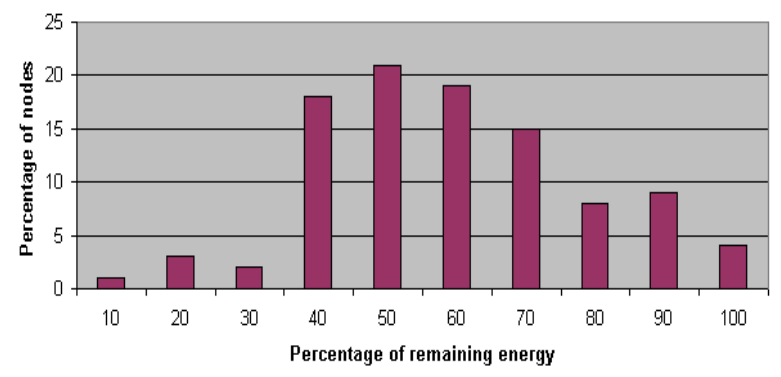

Figure 7. Energy distribution in nodes running TLR

We note from figure 6 that the percentage of remaining energy in the nodes running OLSR is not distributed optimally throughout the network. On one hand, we notice that a high percentage of the nodes hold a high percentage of their initial energy which means they were not actively involved in the routing process. This might seem to be a positive feature but it comes at a cost. Namely, another high percentage of nodes have little remaining energy. Only a few nodes have moderate consumption of their energy during the simulation.

This distribution is not desirable because it shows that around $46 \%$ of the nodes had energy levels of $30 \%$ or less.

A more desirable distribution of energy is shown in figure 7. In this figure it is evident that a very small percentage of nodes have $30 \%$ of less of their initial energy. Also, the percentage of nodes with the higher energy reserves, $80 \%$ and above, is smaller than what was shown in the case of OLSR. Most of the energy is distributed among the nodes equally which leads to an extended lifetime of the nodes in the network.

The second objective of the simulation was to study the average energy reserves of all the nodes in the network throughout the period of the simulation. This was done by using the energy levels given in the trace files of NS2. The sampling of the energy was done in 10 second intervals.

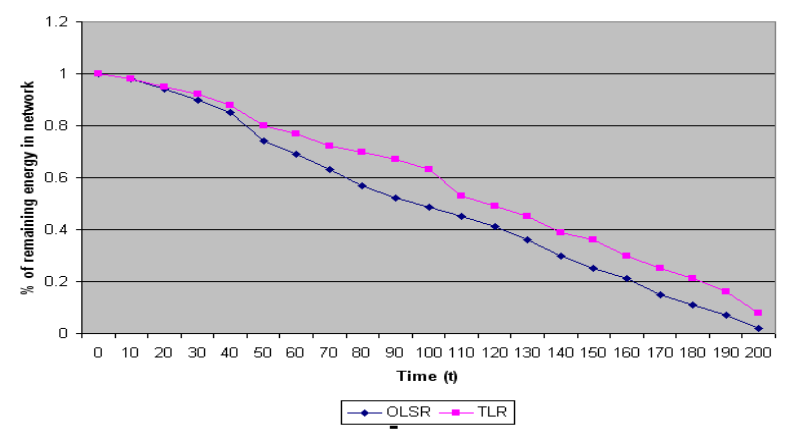

Figure 8. Energy distribution in nodes running OLSR

From figure 8, it is evident that TLR leads to a higher average percentage of remaining energy. This is attributed to the decrease in dropped packets in the network. We see that TLR will give a longer lifetime of the network. 
International Journal of Wireless \& Mobile Networks (IJWMN) Vol. 3, No. 2, April 2011

\section{Conclusions}

Two link state routing protocols were compared in terms of survivability and reliability. Markov models were used to find the reliability function and it was shown how the reliability of TLR is higher than that of OLSR.

The results of this work show the advantage of using TLR instead of OLSR. Notably, TLR results in an extended lifetime of the nodes which may translate to a higher availability of paths.

Future extension of this work includes the analysis of the routing protocols in the case of mobility. In such a case, the intermediate node may move in and out of the communication range.

\section{REFERENCES}

[1] Chen, D., Garg, S., and Trivedi, K. Network Survivability Performance Evaluation: A Quantitative Approach with Applications in Wireless Ad Hoc Networks. In Proceedings of the Fifth ACM International Workshop on Modeling, Analysis and Simulation of Wireless and Mobile Systems, Atlanta, USA, 2002

[2] Chen, H., Mineno, H. and Mizuno, T. An Energy-Aware Routing Scheme with Node Relay Willingness in Wireless Sensor Networks. In Proceedings of the First International Conference on Innovative Computing, Beijing, China, 2006.

[3] Domingo, M., Remondo, D. and Leon, O. A simple Routing Scheme for Improving Ad Hoc Network Survivability. In Proceedings of IEEE GLOBECOM'03, San Francisco, USA, December 1-5, 2003, 718-723.

[4] Jacquet, P. Muhlethaler, P. Clausen, T. Laouiti, A. Qayyum, A., and Viennot, L. Optimized Link State Routing Protocol for Ad Hoc Networks. In Proceedings of the IEEE International Multi Topic Conference on Technology for the 21st Century, (INMIC'01), December, 2001, 62 - 68.

[5] Regatte, N., and Sarangapani, J. Optimized Energy-Delay Routing in Ad Hoc Wireless Networks. In Proceedings of World Wireless Conference, San Francisco, USA. May, 2005.

[6] Singh, S. and Raghavendra, C.S. PAMAS - Power Aware Multi-Access Protocol with Signaling for Ad Hoc Networks. ACM Computer Communications Review,28, 3 (July 1998), 5 - 26.

[7] Taqieddin, E., Sarangapani, J. and Miller, A. Optimal Energy-Delay Routing Protocol with Trust levels for Wireless Ad Hoc Networks. In Proceedings of International Telemetry Conference,(ITC'05), Las Vegas, Nevada, Oct 2005.

[8] Toh, C-K, Cobb, H., and Scott, D. Performance Evaluation of Battery-Life-Aware Routing Schemes for Wireless Ad Hoc Networks. In Proceedings of IEEE International Conference on Communications (IEEE ICC), Helsinki, Finland, June 2001.

[9] Trajanov, D., Filiposka, S., Efnuseva, M. and Grnarov, A. Ad Hoc Networks Connection Availability Modeling. In Proceedings of the $1^{\text {st }}$ International ACM Workshop on Performance Evaluation of Wireless Ad Hoc, Sensor, and Ubiquitous Networks, Venezia, Italy, 2004, 56 - 60.

[10] Zhou, Y., Laurenson, D., and McLaughlin, S. High Survival Probability Routing in Power-aware Mobile Ad Hoc Networks. IEE Electronics Letters, Vol. 40, No. 22, (Oct. 2004), 1424-1426. 